\title{
Improving dispersal of therapeutic nanoparticles in the human body
}

\author{
Tania Limongi ${ }^{1}$, Marta Canta1, Luisa Racca ${ }^{1}$, Andrea Ancona ${ }^{1}$, Stefania Tritta², Veronica \\ Vighetto $^{1}$ \& Valentina Cauda*,1 \\ 1Department of Applied Science \& Technology, Politecnico di Torino, Corso Duca degli Abruzzi 24, Torino 10129, Italy \\ ${ }^{2}$ Department of Molecular Biotechnology and Health Sciences, Molecular Biotechnology Center, University of Torino, Via Nizza 52, \\ Torino 10126, Italy \\ *Author for correspondence: Tel.: +39 011090 7389; valentina.cauda@polito.it, web page: http://areeweb.polito.it/TNHlab/
}

\author{
"the main challenge is to achieve well-dispersed NPs"
}

First draft submitted: 14 February 2019; Accepted for publication: 20 February 2019; Published online: 11 March 2019

Keywords: colloidal stability $\bullet$ hydrodynamic size $\bullet$ in vivo tests $\bullet$ lipid bilayers $\bullet$ organic coating $\bullet$ protein corona $\bullet$ stealth nanoparticles • thrombogenicity

According to the American Society for Testing and Materials, nanoparticles (NPs) are characterized by having two or more dimensions at the nanometer scale and distinctive improved physical/chemical properties compared with their bulk counterpart. Considering their composition, NPs are usually divided into three principal categories: inorganic (metals, metal oxide, ceramic and quantum dots), organic (polymers and lipid-based assembles) and carbon-based NPs [1]. Nano-sized particles exhibit distinctive, chemical and physical properties that denote them as unique nanodevices usable in a wide range of pharmaceutical/therapeutic applications for their capability to affect directly cell vitality or for their drug delivery attitude [2]. They are playing a strategic role in the implementation of new therapeutic solutions against Parkinson's, Alzheimer and infectious diseases, as well as against cancer and many other disorders [3,4]. Usually, the main challenge is to achieve well-dispersed NPs, even in a colloidal manner, in order to facilitate their use both in in vitro and in vivo tests, in other words, for internalization, vitality, differentiation assays and so on. It is therefore required to oversee their size and shape and hence avoid aggregation and agglomeration in body fluids or in cell culture media, both containing proteins, electrolytes, lipids and other biomolecules. As soon as the NPs come into contact with biological fluids, their surface adsorbs a wide range of biomolecules, forming a protein corona which is assumed to comprise a hard layer and a soft one. The former is closely associated with the NP, while the soft layer consists of loosely bound proteins. Many parameters affect NPs' protein corona composition and subsequently their aggregation state and, ultimately, their therapeutic applicability. The presence of a protein corona on NPs dispersed in a biological fluid is unanimously recognized, as well as the fact that it can hugely affect their size, shape and charge [5].

By controlling and measuring the NPs' colloidal stability and aggregation, researchers can have a clear idea of how these factors affect biodistribution, toxicity and pharmacokinetic parameters. Until now different solutions have been implemented to escape the agglomerates' formation in physiological solutions and media.

Nanoparticle dispersion in aqueous media can be described by using the Deryagin, Landau, Verwey and Overbeek (DLVO) theory which takes into account the Van der Waals attractive forces and the electrostatic interactions, allowing assessment of the potential barrier between particles.

In this commentary, we will analyze the role of various dispersant enhancers, including chemical, biological and physical approaches. Actually, dispersion stabilizers as a chemical modification of the NP surfaces are routinely applied at the preparation step of the nanomaterials. Such chemical functionalization includes the use of organic coatings, such as various natural and synthetic polymeric layers. Biologically relevant approaches rely on the use of proteins and lipid bilayers, both from synthetic or natural origins, to shield NPs against aggregation in a biologically relevant environment. For physical methods, the application of ultrasound as a pretreatment to improve NP dispersion is applied regularly for deagglomeration of NPs into biological media. 


\section{Chemical approaches to improve NP dispersion}

In general, the most manageable strategy consists on the stabilization through NPs surface chemistry modification by using PEG coatings, zwitterionic ligands, lipid bilayers, proteins, glycans, mercaptoalkyl acid ligands and/or aptamers.

A common practice to guarantee efficient NPs dispersion time is the so called 'PEGylation' strategy. The use of PEG to coat NPs has many advantages, such as reduced aggregation, phagocytosis and opsonization, which therefore decrease NPs immunogenicity and improve blood half-life, in other words, improved systemic circulation. PEG coating on NPs reduces their nonspecific adsorption of serum proteins and their uptake by the mononuclear phagocytic system [6]. Even if proteins can interact with the PEG coatings, the recognition systems fail to identify the underlying NPs as foreign bodies; in fact, PEG-modification of liposomes considerably increases NPs' circulation times. The first PEGylated nanoparticle product was approved by the US FDA in 1995 for treatment of

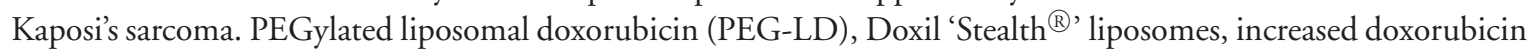
bioavailability by nearly 90 -fold at 1 week from injection versus the free drug, with a drug half-life of $72 \mathrm{~h}$ and a


functional-terminated PEG. As a broadly employed example, thiol-terminated derivatives (PEG-SH) can covalently bind to gold (Au) NPs' surface. In particular, highly dispersed and stable Au NPs in high ionic strength solutions are citrate-stabilized ones; they can be produced by adding a solution of PEG-SH with the desired molecular weight and allowing citrate ligands to gradually exchange with PEG-SH [8]. It should be noted that only sphericalshaped Au NPs can be obtained using citrate buffers, while surfactants as cetyltrimethylammonium bromide $(\mathrm{CTAB})$ are required to define, in high ionic strength solutions, stable anisotropic-shaped nanostructures such as Au nanorods. Methods using PEG-SH ligand exchange reactions have been implemented to replace CTAB with PEG molecules [9]. Considering metal oxide-based NPs, several solutions for guaranteeing an appreciable colloidal stability are again to link functional PEG onto them through a covalent bond or by using silane-terminated PEG [10]. In particular, different silane-terminated PEGs with various molecular weights (from 550 to $5000 \mathrm{Da}$ ) were covalently bound to colloidal mesoporous silica NPs during a sol-gel co-condensation approach. The different PEGylation strategies were then evaluated on the long-term biostability of such mesoporous silica NPs when dispersed in artificial plasma. These experiments demonstrated that longer and denser PEGylated shells are most efficient in preserving the colloidal stability and reducing the biodegradation kinetics of mesoporous NPs in comparison to unfunctionalized ones [10]. Considering another type of nanomaterial, $\mathrm{CdTe} / \mathrm{CdS} / \mathrm{ZnS}$ quantum dots can be directly produced in water by using PEG as the stabilizing agent. Although PEGylation of NPs provides a postsynthetic reproducible functionalization system for enhancing colloidal stability in physiological media, it causes an increase in the NP hydrodynamic size and, especially during in vivo experiments, the host can often develop antibodies against PEG-encapsulated nanocarriers [8].

Zwitterionic ligands, including carboxybetaines and sulfobetaines, containing two or more functional groups with net zero charge, have been described as exceptional ligands for improving NPs' stability in biofluids. Indeed, they customize NPs with smaller hydrodynamic radii and consequently create a lower grade of opsonization. Regrettably, minor changes in $\mathrm{pH}$ can change their behavior, moreover most of them are not yet commercially available and their use involves difficult synthesis procedures.

Mercaptoalkyl acid ligands, such as the 11-mercaptoundecanoic acid (MUA), are also used for NPs surface chemical functionalization. However, it should be noted that they guarantee less colloidal stability then PEG coatings, because they are highly $\mathrm{pH}$-dependent.

Nanoparticles stability has also been enhanced through thiol- or amino-modified aptamers immobilization. Negatively charged aptamer-modified Au NPs remained more stable in high salt solutions as the negative charge inhibits aggregation caused by electrostatic repulsion forces [11]. The principal drawback of aptamer coatings is associated with the low commercial availability of their expensive library.

Glycan add-ons can also modify NPs' properties by enhancing their biocompatibility and solubility. Polysaccharides such as chitosan, dextran, hyaluronic acid and heparin can enable the preparation of a wide range of polysaccharide-based NPs for pharmaceutical and therapeutic applications. Heparin-coated metal oxide NPs, such as colloidal mesoporous silica NPs, have shown improved biostability in blood and efficient antithrombogenic effects, when compared with pristine mesoporous silica NPs [12]. In this example, heparin was covalently bound to the outer surface of amino-functionalized mesoporous silica NPs. The authors claimed that the combination of heparin with the highly porous features of mesoporous NPs can lead to an efficient multifunctional drug-delivery 
system. This smart all-in-one nanosystem can thus act both as an anticoagulant drug, thanks to heparin itself, and as a carrier, in other words, further incorporating other biologically functional molecules or therapeutics as deliverable cargos.

\section{Biological approaches for particle dispersal}

Another strategy to increase the stability of NPs in biological fluids is protein coatings; proteins are stable under physiological conditions through their multitude of charged groups, able to endow NPs with steric and electrostatic stabilization. The protein coating on NPs precludes the adhesion of other proteins by causing a hydration shell through hydrogen bonds with surrounding water molecules. The main concern related with protein coating strategy is the increase in the hydrodynamic diameter of the final NPs.

Additional approaches, inspired by natural materials and processes, concern the thermodynamic and physiological aspects of cell membranes. Indeed, the use of synthetic and biologically derived lipid bilayers and membranes as coating agents allows stabilization of NPs in biological media and human fluids. Lipid bilayers have been successfully used to produce highly homogeneous thin envelopes on NPs surface [13], representing a valid tool for NP delivery in biological fluids thanks to their resemblance to biological membranes. Usually, a lipid film formation represents the first step of NPs lipid bilayer coating; lipids such as cholesterol (Chol), dioleoylphosphatidylcholine (DOPC), 1,2-dimyristoyl-sn-glycero-3-phosphocholine (DMPC) and egg sphingomyelin (ESM) are run together in a chloroform/methanol mixture, dried under a stream of argon and subjected to a vacuum to remove any trace of residual solvent. For the encapsulation, multilamellar vesicles are obtained when NPs are added and sonicated in the lipid films previously hydrated with water; additional sonication are required to reduce the multilamellar vesicles into smaller unilamellar ones containing NPs. In another encapsulation approach, a process called 'solvent exchange' method is proposed. The NPs are dispersed in a solvent solution of free-floating lipids and then the water concentration is promptly increased, leading to the formation of lipid bilayers supported on the NP surface [14,15]. The stability and the long circulating capability of liposomes are still debated along with the fact that their circulation could cause some side effects. It is therefore believed that liposome-encapsulated NPs can guarantee a higher and prolonged stabilization with respect to pristine liposomes and have the advantage of being able to carry a higher amount of therapeutic cargo, in other words, drugs or genes, when highly porous NPs are concerned [14].

In a context of translation toward personalized medicine, extracellular vesicles (EVs) such as exosomes, could be a better choice in terms of bio- and hemo-compatibility, lower immunogenic content, in case of autologous EVs, and, probably, higher dispersion in human biological fluid than artificial liposomes. Examples of NP encapsulation strategies in exosomes were thus proposed $[16,17]$, with the main aim of improving the delivery of anticancer drugs to cell cultures in vitro. In any case, it must be emphasized that the absence of covalent binding onto NP surfaces can be the cause of lipid detachment, and, although lipid coatings stabilize NPs in different biological media, some NP aggregation phenomena have been observed when dispersed in media characterized by high concentrations of cysteine or glutathione [8]. Furthermore, NPs encapsulated into EVs can raise some doubts in terms of encapsulation efficiency, as well as when high yield of the final hybrid materials and low cost-related productions are desired.

\section{Physical dispersion methods}

Prior to performing in vitro or in vivo tests, dry powders or suspensions of NPs must be dispersed in the final medium while maintaining their nanometric size. When dried, NPs are bound together into micro/macrometric structures by both weak physical Van Der Waals forces and strong chemical bonds. Moreover, the high surface area to volume ratio of nanometric particles further increases the interparticle physical attractive forces. To break down and disrupt NP agglomerates, the application of high frequency pressure waves, commonly referred to as 'sonication', is one of the most widely used techniques. Sonication relies in the application of pressure waves at a frequency higher than $20 \mathrm{KHz}$ to the NP suspension to disrupt the particle agglomerates through a process called inertial cavitation. Inertial cavitation refers to the instable oscillation of gas bubbles in liquid due to the applied acoustic pressure. Indeed, when a gas bubble is exposed to an alternating acoustic field, it expands and contracts following the external pressure. When the applied acoustic pressure is higher than the cavitation threshold, the oscillation of the bubble becomes unstable and it violently collapses, generating shock waves and high temperatures at the center of the collapse [18]. The cavitation-induced mechanical effects, such as localized high pressures and shock waves, are the main mechanisms behind NP dispersion by ultrasound. Ultrasound disruption is more energy-efficient and can achieve a higher degree of NP dispersion and optimal deagglomeration, at constant input energy, than other conventional dispersion techniques. Moreover, ultrasound dispersion is a convenient, relatively inexpensive tool 
that is simple to operate and maintain [19]. Furthermore, there are evidences that ultrasound application to NP suspension preceding the addition of a dispersion stabilizer can be more effective than sonication afterward. In a comparison study on titania $\left(\mathrm{TiO}_{2}\right) \mathrm{NP}$ dispersions, two different mechanical dispersion techniques were applied: ultrasonic irradiation and milling with micrometer beads. Ultrasound irradiation resulted in lower aggregate size than milling, thus concluding that ultrasonic dispersion method was a useful way to prepare concentrated and highly dispersed NP suspensions [20]. Therefore, ultrasonic treatment of NP suspension can be considered as an effective and nonexpensive physical method to obtain dispersed NPs, as part of a multistep approach toward stable $\mathrm{NP}$ suspensions for in vitro and in vivo therapeutic applications.

\section{Conclusion}

In conclusion, it seems clear that for testing the effects of NPs on in vitro cells cultures and their in vivo fate, they have to be effectively dispersed in body fluids such as blood, urine, interstitial fluid and so on. It is evident through this commentary that therapies for cancer, infections such as HIV, or for other chronic and acute illnesses, have strongly benefited from the use of nanotechnology tools. The nanomedicine approach thus broadly benefits from improving the dissolution mechanisms of drugs via NP formulation. By tuning NP size and surface properties, researchers and physicians could have a clear indication of how these factors affect the NP biodistribution and they will certainly increase their chances of optimizing drug stability by decreasing their clearance and potential side effects in nanomedical approaches.

Financial \& competing interests disclosure

This work has received funding from the European Research Council (ERC) under the European Union's Horizon 2020 research and innovation program (Grant Agreement No 678151 - Project Acronym "TROJANANOHORSE" - ERC Starting Grant). The authors have no other relevant affiliations or financial involvement with any organization or entity with a financial interest in or financial conflict with the subject matter or materials discussed in the manuscript apart from those disclosed.

No writing assistance was utilized in the production of this manuscript.

Open access

This work is licensed under the Attribution-NonCommercial-NoDerivatives 4.0 Unported License. To view a copy of this license, visit http://creativecommons.org/licenses/by-nc-nd/4.0/

\section{References}

Papers of special note have been highlighted as: • of interest; $\bullet \bullet$ of considerable interest

1. Mauricio MD, Guerra-Ojeda S, Marchio P et al. Nanoparticles in medicine: a focus on vascular oxidative stress. Oxid. Med. Cell. Longevity 2018, 6231482 (2018).

2. Zhang L, Gu F, Chan J, Wang A, Langer R, Farokhzad O. Nanoparticles in medicine: therapeutic applications and developments. Clin. Pharmacol. Ther. 83(5), 761-769 (2008).

3. Torres-Ortega PV, Saludas L, Hanafy AS, Garbayo E, Blanco-Prieto MJ. Micro- and nanotechnology approaches to improve Parkinson's disease therapy. J. Control. Rel. 295, 201-213 (2019).

4. Kevadiya BD, Ottemann BM, Thomas MB et al. Neurotheranostics as personalized medicines. Adv. Drug Deliv. Rev. (2018).

5. Oh JY, Kim HS, Palanikumar L et al. Cloaking nanoparticles with protein corona shield for targeted drug delivery. Nat. Commun. 9(1), 4548 (2018).

- Presents a targeting system in which nanoparticles (NPs) are supramolecularly precoated with a protein corona shield able to reduce serum protein absorption while retaining targeting specificity.

6. Hu CM, Fang RH, Luk BT, Zhang L. Polymeric nanotherapeutics: clinical development and advances in stealth functionalization strategies. Nanoscale 6(1), 65-75 (2014).

7. Bulbake U, Doppalapudi S, Kommineni N, Khan W. Liposomal formulations in clinical use: an updated review. Pharmaceutics 9 (2), 12 (2017).

8. Guerrini L, Alvarez-Puebla RA, Pazos-Perez N. Surface modifications of nanoparticles for stability in biological fluids. Materials 11(7), 1154 (2018).

- Reviews in a very detailed way the latest strategies to achieve stable NPs dispersions in biological fluids.

9. Liu K, Zheng Y, Lu X et al. Biocompatible gold nanorods: one-step surface functionalization, highly colloidal stability, and low cytotoxicity. Langmuir 31(17), 4973-4980 (2015).

10. Cauda V, Argyo C, Bein T. Impact of different PEGylation patterns on the long-term bio-stability of colloidal mesoporous silica nanoparticles. J. Mater. Chem. 20(39), 8693-8699 (2010). 
11. Urmann K, Modrejewski J, Scheper T, Walter Johanna G. Aptamer-modified nanomaterials: principles and applications. BioNanoMaterials 18(1-2), (2017).

12. Argyo C, Cauda V, Engelke H, Rädler J, Bein G, Bein T. Heparin-coated colloidal mesoporous silica nanoparticles efficiently bind to antithrombin as an anticoagulant drug-delivery system. Chem. Eur. J. 18(2), 428-432 (2012).

- Reports for the first time the shielding of a NP with heparin (Hep), showing that the same hemocompatible and antithrobogenic properties of Hep can be translated to the NP when Hep is bound to its surface.

13. Chakraborty S, Abbasi A, Bothun GD, Nagao M, Kitchens CL. Phospholipid bilayer softening due to hydrophobic gold nanoparticle inclusions. Langmuir 34(44), 13416-13425 (2018).

14. Cauda V, Engelke H, Sauer A et al. Colchicine-loaded lipid bilayer-coated $50 \mathrm{~nm}$ mesoporous nanoparticles efficiently induce microtubule depolymerization upon cell uptake. Nano Lett. 10(7), 2484-2492 (2010).

15. Dumontel B, Canta M, Engelke $\mathrm{H}$ et al. Enhanced biostability and cellular uptake of zinc oxide nanocrystals shielded with a phospholipid bilayer. J. Mater. Chem. B 5(44), 8799-8813 (2017).

16. Illes B, Hirschle P, Barnert S, Cauda V, Wuttke S, Engelke H. Exosome-coated metal-organic framework nanoparticles: an efficient drug delivery platform. Chem. Mater. 29(19), 8042-8046 (2017).

17. Srivastava A, Amreddy N, Babu A et al. Nanosomes carrying doxorubicin exhibit potent anticancer activity against human lung cancer cells. Sci. Rep. 6, 38541 (2016).

- This paper interestingly shows for the first time the insertion of gold NPs into exosomes as an innnovative and more efficient drug carrier for lung cancer therapy.

18. Canavese G, Ancona A, Racca L et al. Nanoparticle-assisted ultrasound: a special focus on sonodynamic therapy against cancer. Chem. Eng. J. 340, 155-172 (2018).

- Highlights the role of ultrasound-based therapies based on the use of NP and especially opening new horizons in the oncological field.

19. Mandzy N, Grulke E, Druffel TB. Breakage of $\mathrm{TiO}_{2}$ agglomerates in electrostatically stabilized aqueous dispersions. Powder Technol. 160, 121-126 (2005).

20. Sato K, Li J-G, Kamiya H, Ishigaki T. Ultrasonic dispersion of $\mathrm{TiO}_{2}$ nanoparticles in aqueous suspension. J. Am. Ceram. Soc. 91(8), 2481-2487 (2008). 